\title{
711 IGM-7354 IS AN ANTI-PD-L1 IGM ANTIBODY AND IL-15 CYTOKINE FUSION THAT ENHANCES NK AND CD8+ T CELL PROLIFERATION AND TUMOR CYTOTOXICITY PLUS POTENTLY REVERSES T CELL EXHAUSTION
}

Thierry Giffon*, Melanie Desbois, Dean Ng, Poonam Yakkundi, Marigold Manlusoc Miho Oyasu, Rodnie Rosete, Daniel Machado, Susan Calhoun, Tasnim Kothambawala, Avneesh Saini, Beatrice Wang, Maya Kotturi, Bruce Keyt, Angus Sinclair. IGM Biosciences Inc, Mountain View, CA, United States

Background While approved PD-1/PD-L1 inhibitory antibodies have demonstrated clinical efficacy in certain cancer patients, relapse following a primary response is often observed. Enhancing anti-tumor immune responses with an immunostimulatory cytokine, IL-15 is an attractive combination strategy to enhance anti-tumor NK and memory CD8 + T cell expansion and survival. We have developed IGM-7354, a high affinity, high avidity anti-PD-L1 pentameric IgM antibody with an IL-15R $\alpha$ chain and IL-15 fused to the joining (J) chain, designed to deliver IL-15 to PD-L1 expressing tumors for enhancing anti-tumor immune responses.

Methods IGM-7354 was generated by grafting heavy chain variable regions of a high affinity humanized anti-PD-L1 IgG onto the IgM heavy chain framework, co-expressed with the light chain and the $\mathrm{J}$ chain which included a single IL-15R $\alpha$ and IL-15 fusion. Binding ELISAs were performed using recombinant antigens. Human and cynomolgus monkey PBMCs were used for potency testing. Reversal of $\mathrm{T}$ cell exhaustion was tested using in vitro MLR. In vitro cytotoxicity assays were performed with luciferase-tagged MDA-MB231 cells and PBMCs. In vivo pharmacodynamic studies were conducted in mice and cynomolgus monkeys.

Results IGM-7354 bound human and cynomolgus monkey PDL1 with the same affinity but did not bind to rat or mouse PD-L1. In addition, the IL-15 component of IGM-7354 bound to human and cynomolgus $\beta$ chain of the trimeric IL-15 receptor with similar affinities, but with weaker binding affinity to rodent IL-15R $\beta$. Using in vitro assays with PBMCs, IGM-7354 dose dependently enhanced the proliferation of human and cynomolgus monkey NK and CD8 + T cells. Furthermore, IGM-7354 was able to reverse $\mathrm{T}$ cell exhaustion in an in vitro MLR beyond that of an IL-15/IL15R $\alpha$ complex or anti-PD-L1 IgM or IgG alone, as demonstrated by an increase in activation and effector cytokine secretion. IGM-7354 also enhanced in vitro killing of PD-L1-expressing MDA-MB-231 breast cancer cells by human PBMCs. Pharmacodynamic studies in an MDA-MB-231 xenograft mouse model showed dosedependent increases in circulating $\mathrm{NK}$ and $\mathrm{CD} 8+\mathrm{T}$ cells and tumor infiltrating lymphocytes, which correlated with tumor regression. In cynomolgus monkeys, intravenous administration of IGM-7354 was well tolerated and dose dependently induced the proliferation of $\mathrm{NK}$ and $\mathrm{CD} 8+\mathrm{T}$ cells.

Conclusions IGM-7354 stimulates $\mathrm{NK}$ and CD8 $+\mathrm{T}$ cell expansion in vitro and in vivo plus induces tumor regressions in mouse tumor models. This approach may enhance tumor localization of the immunostimulatory cytokine IL-15 through high affinity and high avidity binding to PD-L1 thereby improving anti-tumor responses and minimizing toxicity.

Ethics Approval All animal studies were conducted according to approved Institutional Animal Care and Use Committee (IACUC) protocols of the testing facilities.

http://dx.doi.org/10.1136/jitc-2021-SITC2021.711 